\title{
Speeding Disease Gene Discovery with SUSPECTS
} Euan Adie*

\author{
Address: Medical Genetics Section, School of Molecular and Clincal Medicine, University of Edinburgh, UK \\ Email: Euan Adie* - Euan.adie@ed.ac.uk \\ * Corresponding author
}

from BioSysBio: Bioinformatics and Systems Biology Conference

Edinburgh, UK, I4-15 July 2005

Published: 2I September 2005

BMC Bioinformatics 2005, 6(Suppl 3):PI

The genomic regions identified through genetic linkage studies of complex disease are frequently large and may contain hundreds of genes. This number can be reduced by the candidate gene approach - matching functional annotation to what is known about the etiology of the disease in question.

SUSPECTS is a web based tool designed to help researchers prioritize genes in a region of interest. It is based on the assumption that the genes underlying a particular complex disease tend to share the same or similar biological pathways. The Gene Ontology annotation, Interpro domains, expression profiles and sequence features of each gene in the region are compared with a training set of genes already implicated in the disease. Genes are automatically assigned a score on the basis of the number of different lines of evidence where a match was found and the significance of those matches; this score reflects the relative probability of the gene's involvement in the disease under study.

As a case study we ran SUSPECTS over the entire human genome with a training set of genes implicated in schizophrenia. By searching PubMed we found plausible evidence of association with schizophrenia for around half of the top 25 candidates selected; these included serotonin, dopamine and GABA-B receptor subunits as well as less obvious candidates like PER1 (Period Circadian Protein 1). 